\title{
A Sequence-based Antibody Paratope Prediction Model Through Combing Local-Global Information and Partner Features
}

\author{
Shuai Lu, Yuguang Li, Xiaofei Nan*, and Shoutao Zhang*
}

\begin{abstract}
Antibodies are proteins which play a vital role in the immune system by recognizing and neutralizing antigens. The region on the antibody binding to an antigen, known as paratope, mediates antibody-antigen interaction with high affinity and specificity. And the accurate prediction of those regions from antibody sequence contributes to the design of therapeutic antibodies and remains challenging. However, the experimental methods are time-consuming and expensive. In this article, we propose a sequence-based method for antibody paratope prediction by combing local and global information of antibody sequence and partner features from partner antigen sequence. Convolution Neural Networks(CNNs) and a sliding window approach on antibody sequence are used to extract local information. Attention-based Bidirectional Long Short-Term Memory (Att-BLSTM) on antibody sequence are used to extract global information. Also, the partner antigen is vital for paratope prediction, and we employ Att-BLSTM on the partner antigen sequence as well. The outputs of CNNs and Att-BLSTM networks are combined to predict antibody paratope by fully-connected networks. The experiments show that our proposed method achieves superior performance over the state-of-the-art sequenced-based antibody paratope prediction methods on benchmark datasets.
\end{abstract}

Index Terms-CNN, Bi-LSTM, attention, paratope prediction

\section{INTRODUCTION}

$\Lambda$ NTIBODIES play an import role in human immune system by binding a wide range of macromolecules with high affinity and specificity[1]. This ability of binding is mediated by the interaction of amino acid residues at the paratope region of antibody[2]. Accurate prediction of antibody paratope is helpful for the study of antibodyantigen interaction mechanism and the development of antibody design[3]-[5]. Along with the establishment of antibody expression and purification process, the number of approved therapeutic antibodies is growing steadily[6]. As a result, correct judgement of which amino acid residues belonging to paratope is essential[7].

The antibody paratope region is usually determined by observing the residues that are spatially close to its partner antigen from its structure obtained by X-ray[8], NRM[9] and Cryo-EM[10]. However, these experimental methods are time-consuming and expensive[11]. Therefore, a lot of computational models are developed to overcome these problems. According to the input features, the computational methods for antibody paratope prediction can be classified into two classes: sequence-based and structure-based. As the name implies, the sequence-based models only use

- Shuai Lu and Xiaofei Nan are with School of Information Engineering, Zhengzhou University, Zhengzhou, Henan, 450001, China. E-mail: ielushuai@126.com, iexfnan@zzu.edu.cn.

- Yuguang Li is with College of Economics and Management, Zhengzhou University of Light Industry, Zhengzhou, Henan, 450001, China. E-mail:liyuguang1993@163.com

- Shoutao Zhang is with School of Life Science, Zhengzhou University, Zhengzhou, Henan, 450001, China.

E-mail: zhangst@zzu.edu.cn

- *Corresponding authros: Xiaofei Nan(iexfnan@zzu.edu.cn) and Shoutao Zhang(zhangst@zzu.edu.cn) the antibody sequence features and the structure-based models utilize the antibody structure features as well. Some of the sequence-based methods take the whole antibody primary sequence as input and output the binding probability of each amino acid residue[12]-[14]. Other methods only utilize the residues in complementarity determining regions (CDRs) and predict their binding probability[15], [16]. However, about $20 \%$ of the residues that participating in binding fall outside the CDRs[17]. Most structurebased methods consider antibody structure as a graph and employ graph convolution operation for aggregating the information of spatial neighboring residues[18]-[20]. Apart from those methods, 3D Zernike descriptors are used for representing a set of amino acid residues adjacent to each other, and shallow machine learning models are used[21]. Although structure-based models can provide more accurate description of paratope, sequence information is always available earlier than structure[15].

Global information of the whole sequence has been proved useful in many biology biological analysis tasks such as protein-protein interaction sites prediction[22] and protein phosphorylation sites prediction[23]. Methods from those works extract global features form the whole protein sequence by TextCNNs[24] or SENet blocks[25] and BiLSTM blocks[26]. In this study, we utilize Attention-based Bidirectional Long Short-Term Memory(Att-BLSTM) to extract global features from antibody sequence and partner features form its partner antigen sequence, as Att-BLSTM shows superior performance in several machine learning tasks[27], [28].

In this work, we propose a sequence-based method for antibody paratope prediction utilizing both local-global information and partner features by combing Convolutional 
Neural Networks (CNNs) and Att-BLSTM networks. For local features, we use CNNs with a fixed sliding window size to consider the neighbor information around a target amino acid residue on antibody sequence. For global features and partner features, we use Att-BLSTM networks on antibody sequence and its partner antigen sequence, respectively. After that, all features are combined to fed into fully-connected networks to predict the probability of each antibody residue belonging to paratope. We also compare results with other competing sequence-based paratope predictors, and our method achieves the best performances.

\section{Materials AND MEthods}

\subsection{Datasets}

The datasets used in this study are taken from PECAN[20]. All complexes are filtered to make sure that no antibody sequence share identity more than $95 \%$. The complexes in training and validation sets are collected from the training sets of other paratope prediction works[12], [15], [29] and $\mathrm{AbDb}[30]$. The training set contains 205 complexes and the validation set consists of 103 complexes to tune the hyper parameters in our model. And, 152 complexes are used for testing and evaluating the performance of our model.

Similar as other works, a residue on antibody is defined to belong to paratope if any of its heavy atoms is less than $4.5 \AA$ away from any heavy atom on antigen[15], [20], [29]. It should be noted that the structure of antibody and antigen complex is used only extracted positive and navigate labels of antibody residues. The summary of datasets is shown in Table 1.

TABLE 1

Number of complexes and residues in the datasets.

\begin{tabular}{cccc}
\hline DataSet & Complexes & Positive residues & Negative residues \\
\hline Training & 205 & $4449(5.19 \%)$ & $81283(94.81 \%)$ \\
Validation & 103 & $2237(5.24 \%)$ & $60584(94.76 \%)$ \\
Testing & 152 & $3314(5.19 \%)$ & $40480(94.81 \%)$ \\
\hline
\end{tabular}

\subsection{Input features}

We utilize residue features consist of one-hot encoding, evolutionary information, seven additional parameters and predicted structural features of antibody or antigen sequence. All of them are described in details as follows:

\subsubsection{One-hot encoding of antibody sequence}

Only 20 natural types of amino acid residues are considered in our study. Each type is encoded to a 20D vector where each element is either 1 or 0 and 1 indicates the existence of a corresponding amino acid residue.

\subsubsection{Evolutionary information}

A lot of studies use the evolutionary information of antibody or protein sequence for biological analysis tasks, such as B-cell epitope prediction[31], [32], protein-protein interaction sites prediction[22], [33]-[36], protein-DNA binding residues prediction[37], protein folding recognition[38] and protein contact map prediction[39]. In this study, we run
PSI-BLAST[40] against the nonredundant (nr)[41] database for every antibody and antigen sequence in our datasets with three iterations and an E-value threshold of 0.001. After that, we get the position-specific scoring matrix (PSSM) and the position- specific frequency matrix (PSFM). Each amino acid is encoded as a $20 \mathrm{D}$ vector representing the probabilities and frequencies of 20 natural amino acid residues occurring at each position in PSSM and PSFM, respectively. For each protein sequence with $\mathrm{L}$ residues, there are $\mathrm{L}$ rows and 20 columns in PSSM or PSFM. Besides PSSM and PSFM, two parameters at each residue position are obtained as well. One is related with column entropy in multiple sequence alignment, and another is related with column gaps in multiple sequence alignment.

\subsubsection{Seven additional parameters}

Those parameters represent physical, chemical and structural properties of each type of amino acid residue by training artificial neural networks for protein secondary structure prediction[42].

\subsubsection{Predicted structural features}

In this study, we predict antibody or antigen local structural features from sequence by NetSurfP-2.0 which is a novel deep learning model trained on several independent datasets[43]. Solvent accessibility, secondary structure, and backbone dihedral angles for each residue of the input sequences are returned from NetSurfP-2.0. Among those features prediction tasks, NetSurfP-2.0 achieves the stateof-the-art performance.

We calculate the absolute and relative solvent accessibility surface accessibility(ASA and RSA, respectively) , 8-class secondary structure classification (SS8), and the backbone dihedral angles $(\phi$ and $\psi$ ) for each residue position of input antibody or antigen sequence. ASA and RSA represent the solvent accessibility of an amino acid residue. The predicted secondary structure describes the local structural environment of a residue. And, $\phi$ and $\psi$ figure the relative positions of adjacent residues. The 8-class secondary structures are: 3-helix (G), a-helix (H), p-helix (I), b-strand (E), b-bridge (B), b-turn (T), bend (S) and loop or irregular (L). And, we use the one-hot encoding of SS8.

Together, an $80 \mathrm{D}$ vector is used for representing a residue in our study.

\subsection{Input representation}

The antibody paratope prediction problem can be summarized as a binary classification task: judging whether a residue from a given antibody sequence binding with its partner antigen or not. As described in Section 2.2, each residue is encoded into an $80 \mathrm{D}$ vector. And each antibody or antigen sequence can be represented as a matrix $S$, including a list of residues:

$$
S=\left[r_{1}, r_{2}, r_{3}, \cdots, r_{i}, \cdots, r_{l}\right]^{T}, S \in R^{(l * d)}
$$

where $r_{i} \in R^{d}$ is the residue feature vector corresponding to the $i$-th residue in the antibody or antigen sequence, $l$ is the sequence length, and $d$ is the residue feature dimen$\operatorname{sion}(80$ in this paper). 


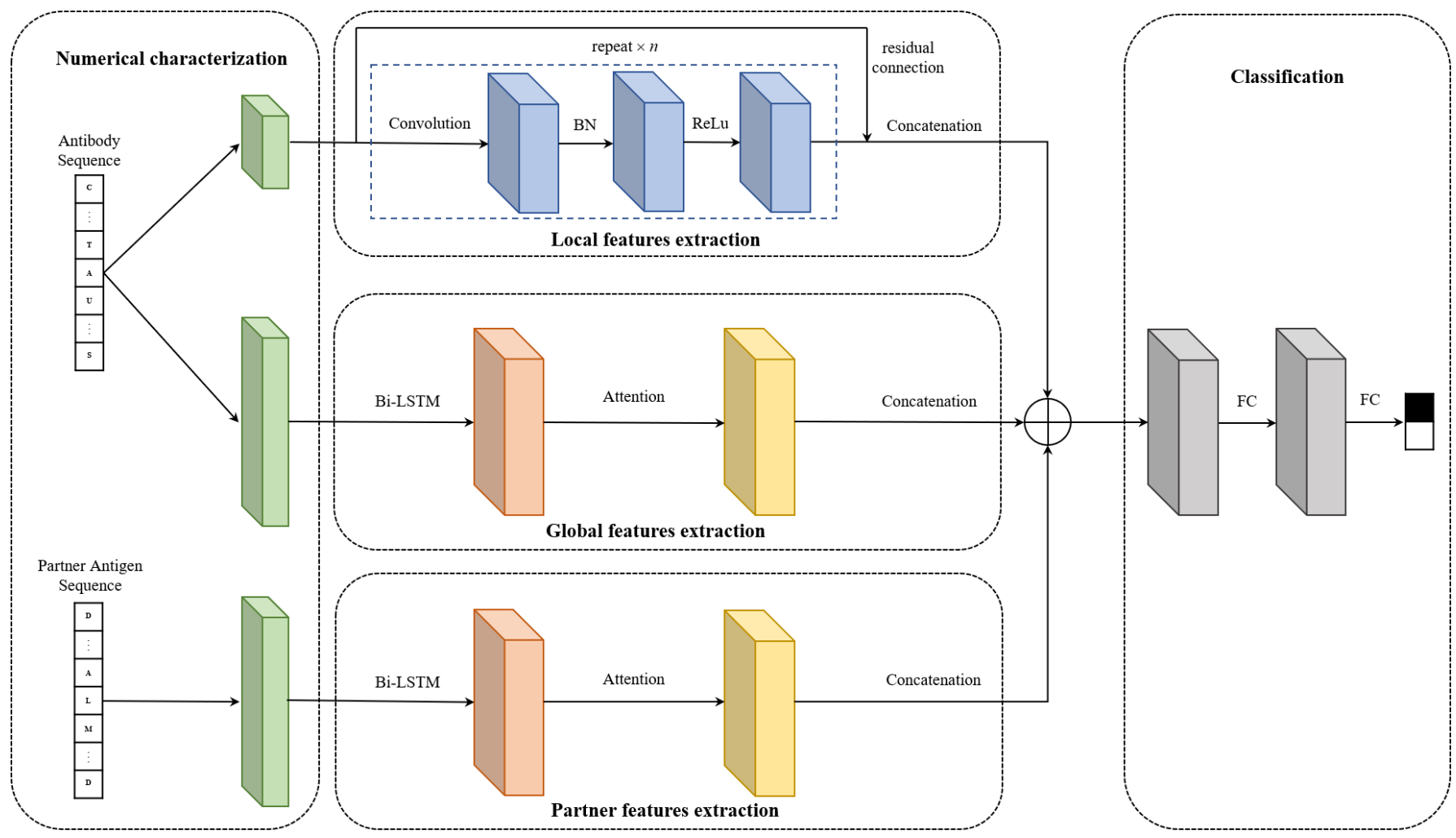

Fig. 1. Architecture of our method

\subsection{Model architecture}

As Fig.1 shows, our proposed method is mainly composed of three parallel parts: CNNs extract local features from antibody sequence, Att-BLSTM networks extract global features from antibody sequence and another Att-BLSTM network extracts partner features from partner antigen sequence. The outputs of those parts are concatenated and fed to fully connected networks to predict the binding probability for each antibody residue.

\subsubsection{CNNs}

Convolution neural networks (CNNs) model has been used in some bioinformatic tools for protein binding site prediction[44], protein-ligand scoring[45] and protein-compound affinity prediction[46]. In our method, the input of CNNs is the local information of the target antibody residue which can be represented as $r_{i-w: i+w}$. It means that we consider the target antibody residue at the center with $2 w$ neighboring residues representing the context of target antibody residue. Those antibody residues which do not have neighboring residues in the left or right are padded by all-zero vectors. The convolutional operation is shown as:

$$
r_{i}^{\prime}=f\left(W_{c} r_{i-w: i+w}+b_{c}\right)
$$

where $f$ is a non-linear activation function (e.g. ReLU), $W_{c}$ is the weight matrix, $r_{i-w: i+w}$ the concatenation of the local information of target antibody residue, and the $b_{c}$ is the bias vector. As Fig. 1 shows, BN means a batch norm layer and the repeat time is 3 in out model. Also, residual connection is used by adding inputs to outputs:

$$
r_{i}^{\prime}=f\left(W_{c} r_{i-w: i+w}+b_{c}\right)+r_{i-w: i+w}
$$

\subsubsection{Att-BLSTM networks}

Attention-based Bidirectional Long Short-Term Memory (Att-BLSTM) networks have been used in chemical and biomedical text processing tasks[47], [48]. However, its advantage has not been exploited in biology sequence analysis such as antibody paratope prediction. In this study, AttBLSTM networks are used to capture global features of antibody and antigen sequences.

As shown in Fig.2, the architecture of Att-BLSTM consists of four parts: input layer, Bi-LSTM layer, attention layer and output layer. The input antibody or antigen sequence is represented as a set of residues: $S=$ $\left[r_{1}, r_{2}, r_{3}, \cdots, r_{i}, \cdots, r_{l}\right]^{T}, S \in R^{(l * d)}$

The Bi-LSTM layer contains two Long Short-Term Memory (LSTM) networks of which one is forward taking input residues form the beginning to the end and another is backward taking input residues from the end to the beginning. A standard LSTM contains three gates and a cell memory state to store and access information over time. Typically, a cell of LSTM can be computed at each time $t$ as follows:

$$
\begin{aligned}
& i_{t}=\sigma\left(W_{i} *\left[h_{t-1}, r_{t}\right]+b_{i}\right) \\
& f_{t}=\sigma\left(W_{f} *\left[h_{t-1}, r_{t}\right]+b_{f}\right) \\
& o_{t}=\sigma\left(W_{o} *\left[h_{t-1}, r_{t}\right]+b_{o}\right)
\end{aligned}
$$




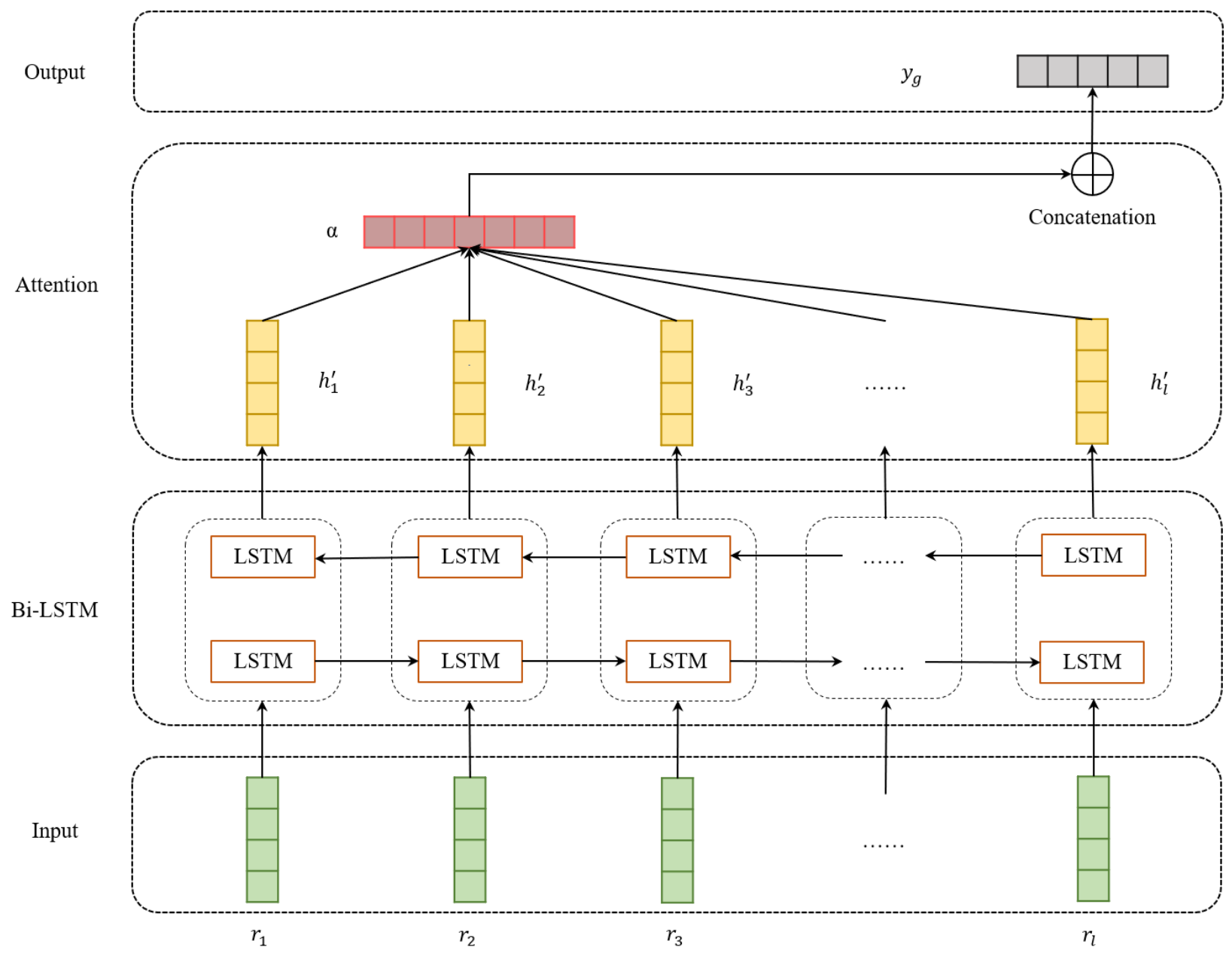

Fig. 2. Architecture of Att-BLSTM

$$
\begin{gathered}
c_{t}=f_{t} * c_{t-1}+i_{t} *\left(\tanh \left(W_{c} *\left[h_{t-1}, r_{t}\right]+b_{c}\right)\right) \\
h_{t}=o_{t} * \tanh \left(c_{t}\right)
\end{gathered}
$$

where $W_{i}, W_{f}, W_{o}$ are the weight matrixs, and $b_{i}, b_{f}$, $b_{o}$ are the biases of input gate, forget gate and output gate, respeatively. And, tanh is the element-wise hyperbolic tangent, $\sigma$ is the logistic sigmoid function, $r_{t}, h_{t-1}$ and $c_{t-1}$ are inputs, and $h_{t}$ and $c_{t}$ are outputs. For the $i-t h$ residue in the input antibody or antigen sequence, we combine the output $\overrightarrow{h_{i}}$ of forward LSTM and output $\overleftarrow{h_{i}}$ of the backward LSTM by concatenating them:

$$
h_{i}^{\prime}=\left[\overrightarrow{h_{i}} \oplus \overleftarrow{h_{i}}\right]
$$

In attention layer, let $H \in R^{(l * 2 d)}$ be a input sequence $\left[h_{1}^{\prime}, h_{2}^{\prime}, h_{3}^{\prime}, \ldots, h_{i}^{\prime}, \ldots, h_{l}^{\prime}\right]^{T}$ in which each element is the $\mathrm{i}$-th residue output of Bi-LSTM, where 1 is the input sequence length, and $d$ is the residue features dimension. The attention mechanism can be calculated as follows:

$$
\begin{gathered}
M=\operatorname{tanth}(H) \\
\alpha=\operatorname{softmax}\left(W_{\mu} M\right)
\end{gathered}
$$

$$
y_{g}=a b_{g} / a g_{g}=H \alpha^{T}
$$

where tanh is the element-wise hyperbolic tangent, $W_{u}$ is the weight matrix, and $\alpha$ is an attention vector. The output $y_{g}\left(a b_{g}\right.$ grepresenting for the output of antibody sequence and $a g_{g}$ representing the output of antigen sequence) is formed by a weighted sum of vectors in $H$.

\subsubsection{Fully-connected networks}

As shown in Fig. 1 and 2, the local features extracted by CNNs is $r_{i}^{\prime}$ and the global features derived by Att-BLSTM networks from antibody and its partner antigen sequence are $a b_{g}$ and $a g_{g}$, respectively. And then, $r_{i}^{\prime}, a b_{g}$ and $a g_{g}$ are concatenated and fed to the fully-connected networks. The calculation of probability $y_{i}$ for each input antibody residue belonging to paratope is shown as:

$$
y_{i}=f\left(W\left(\left(r_{i}^{\prime} \oplus a b_{g} \oplus a g_{g}\right)+b\right)\right.
$$

\subsection{Training details}

We implement our model using PyTorch v1.4. The training configurations are: loss function: weighted cross-entropy loss function as in [49], optimization: Momentum optimizer with Nesterov accelerated gradients; learning rate: 0.001; 
bioRxiv preprint doi: https://doi.org/10.1101/2021.07.17.452531; this version posted July 19, 2021. The copyright holder for this preprint (which was not certified by peer review) is the author/funder, who has granted bioRxiv a license to display the preprint in perpetuity. It is made available under aCC-BY-NC-ND 4.0 International license.

TABLE 2

Performances comparison with competing methods

\begin{tabular}{ccccc}
\hline Method & AUC ROC & AUC PR & MCC & F-score \\
\hline Parapred & $0.880 \pm 0.002$ & - & $0.564 \pm 0.007$ & 0.69 \\
Fast-Parapred & $0.883 \pm 0.001$ & - & $0.572 \pm 0.004$ & - \\
AG-Fast-Parapred & $0.899 \pm 0.004$ & - & $0.598 \pm 0.012$ & - \\
proABC-2 & 0.91 & - & 0.56 & 0.62 \\
Our Method & $\mathbf{0 . 9 4 5} \pm \mathbf{0 . 0 0 1}$ & $\mathbf{0 . 6 4 4} \pm \mathbf{0 . 0 0 2}$ & $\mathbf{0 . 6 3 5 \pm 0 . 0 0 5}$ & $\mathbf{0 . 8 1 9} \pm \mathbf{0 . 0 0 3}$ \\
\hline
\end{tabular}

TABLE 3

Performances of our method using different features combination

\begin{tabular}{ccccc}
\hline Features & AUC ROC & AUC PR & MCC & F-score \\
\hline Local & $0.930 \pm 0.001$ & $0.626 \pm 0.001$ & $0.608 \pm 0.001$ & $0.805 \pm 0.001$ \\
Local + Global & $0.946 \pm 0.001$ & $0.643 \pm 0.002$ & $0.633 \pm 0.009$ & $0.818 \pm 0.005$ \\
Local + Partner & $\mathbf{0 . 9 4 8} \pm \mathbf{0 . 0 0 2}$ & $0.632 \pm 0.012$ & $0.626 \pm 0.018$ & $0.814 \pm 0.009$ \\
Local + Global + Partner & $0.945 \pm 0.001$ & $\mathbf{0 . 6 4 4} \pm \mathbf{0 . 0 0 2}$ & $\mathbf{0 . 6 3 5} \pm \mathbf{0 . 0 0 5}$ & $\mathbf{0 . 8 1 9} \pm \mathbf{0 . 0 0 3}$ \\
\hline
\end{tabular}

batch size: 32; dropout: 0.5; a fixed sliding window length: 11. The first fully connected layer has 512 nodes and the second fully connected layer has 256 nodes. Training time of each epoch varies roughly from 1 to 2 minutes depending on the global features are used or not, using a single NVIDIA RTX2080 GPU.

\section{Results}

\subsection{Model results}

To evaluate the performance of our method and other competing methods, we use the standard metrics, i.e. area under the receiver operating characteristics curve (AUC ROC), the area under the precision recall curve (AUC PR), MCC and F-score. Because our method output a probability for each antibody residue, we compute MCC and F-score by predicting residues with probability above 0.5 as paratope. To summarize the performance, all metrics are averaged over all antibodies in the testing set. We repeat the training and testing procedures five times for providing robust estimates of performance. The mean value and standard error are reported in Table 2.

Table 2 shows the results of our method and other competing sequence-based antibody paratope prediction methods. Parapred uses CNNs and LSTM for predicting probability of the residues in CDRs of antibody sequence[15]. Fast-Parapred and AG-Fast-Parapred outperform Parapred by leveraging self-attention and cross-modal attention, respectively[16]. proABC-2 is based on CNNs model and takes separate heavy chain and light chain as input[14]. The results of proABC-2 is trained on the Parapred-set and uses a threshold of 0.37 . As Table 2 shows, our method performs best on all metrics.

\subsection{Effect of global features and partner features}

Although global features have been proved useful in protein-protein interaction sites prediction [22] and protein phosphorylation sites prediction [23]. Those methods only use global features from self-sequence and don't consider the partner features form the partner-sequence. To measure the effect of global features and partner features, we train our model using different feature combinations. The results are shown in Table 3. Except for AUC ROC, the combination of local-global and partner features achieves best performances on all other metrics. The dataset is imbalanced, and we consider AUC PR as the primary metric because itis more sensitive on an imbalanced dataset[50].

The results from Table 3 indicates that both global and partner features in our method can help for improving the model performance for antibody prediction and the combination of all features performs best. The partner antigen information is also used in AG-Fast-Parapred. AG-FastParapred and Fast-Parapred are from the same work and trained on the same datasets[16].And, Fast-Parapred only utilizes antibody information. From Table 2, we can find that AG-Fast-Parapred perform better than Fast-Parapred which also show the effect of partner features.

\section{Conclusion}

In this study, we proposed a sequence-based antibody paratope prediction method leveraging local and global features of antibody and global features of partner antigen. CNNs model are used to extract the local features of antibody sequence. Att-BLSTM networks are used to capture the global features of the whole antibody and antigen sequence. We implement our model on benchmark datasets and the results show improvement of antibody paratope prediction. Moreover, our results declare that both global features from antibody and antigen are useful for performance improvement and our model performs best when both global features are used together

Though our method outperforms other competing sequence-based methods for antibody paratope prediction, it also has some limitations. First, similar like 
other sequence-base methods, our program takes a lot of time to generate sequence-based features by running PSIBLSAT[40] and NetsurfP-2.0[43]. Second, although the combined features improve the model performance, it is still inferior to the structure-based approaches.

In our work, we show that combing local-global and partner antigen features can be useful for antibody paratope prediction. In the future, we would focus on how to mine more structural features from antibody sequence for improving our model performance.

\section{ACKNOWLEDGMENTS}

This work was supported by Bingtuan Science and Technology Project(2019AB034), 'Created Major New Drugs' of Major National Science and Technology (No. 2019ZX09301159), and Leading Talents Fund in Science and Technology Innovation in Henan Province(194200510002). Xiaofei Nan and Shoutao Zhang are the corresponding authors for this paper.

\section{References}

[1] X. Shen and T. R. Kosten, "Immunotherapy for Drug Abuse," CNS Neurol. Disord. - Drug Targets, vol. 10, no. 8, pp. 876-879, 2012, doi: 10.2174/187152711799219352.

[2] J. W. Stave and K. Lindpaintner, "Antibody and Antigen Contact Residues Define Epitope and Paratope Size and Structure," J. Immunol., vol. 191, no. 3, pp. 1428-1435, Aug. 2013, doi: 10.4049/jimmunol.1203198.

[3] D. Hu et al., "Effective optimization of antibody affinity by phage display integrated with high-throughput DNA synthesis and sequencing technologies," PLoS One, vol. 10, no. 6, pp. 1-17, Jun. 2015, doi: 10.1371/journal.pone.0129125.

[4] A. K. Mishra and R. A. Mariuzza, "Insights into the structural basis of antibody affinity maturation from next-generation sequencing," Frontiers in Immunology, vol. 9, no. FEB. Frontiers Media S.A., pp. 117-127, Feb. 01, 2018, doi: 10.3389/fimmu.2018.00117.

[5] J. O. Zhou, H. A. Zaidi, T. Ton, and D. Fera, "The Effects of Framework Mutations at the Variable Domain Interface on Antibody Affinity Maturation in an HIV-1 Broadly Neutralizing Antibody Lineage," Front. Immunol., vol. 11, pp. 1529-1541, Jul. 2020, doi: 10.3389/ fimmu.2020.01529.

[6] R. M. Lu et al., "Development of therapeutic antibodies for the treatment of diseases," J. Biomed. Sci., vol. 27, no. 1, pp. 1-30, 2020, doi: 10.1186/s12929-019-0592-z.

[7] R. Esmaielbeiki, K. Krawczyk, B. Knapp, J. C. Nebel, and C. M. Deane, "Progress and challenges in predicting protein interfaces," Brief. Bioinform., vol. 17, no. 1, pp. 117-131, 2016, doi: 10.1093/bib/bbv027.

[8] F. Schotte et al., "Watching a protein as it functions with 150-ps time-resolved x-ray crystallography," Science (80-. )., vol. 300, no. 5627, pp. 1944-1947, Jun. 2003, doi: 10.1126/science.1078797.
[9] A. Bax and S. Grzesiek, "Methodological Advances in Protein NMR," Acc. Chem. Res., vol. 26, no. 4, pp. 131-138, Apr. 1993, doi: 10.1021/ar00028a001.

[10] Z. H. Zhou, "Towards atomic resolution structural determination by single-particle cryo-electron microscopy," Current Opinion in Structural Biology, vol. 18, no. 2. pp. 218-228, Apr. 2008, doi: 10.1016/j.sbi.2008.03.004.

[11] D. Kuroda, H. Shirai, M. P. Jacobson, and H. Nakamura, "Computer-aided antibody design," Protein Eng. Des. Sel., vol. 25, no. 10, pp. 507-521, 2012, doi: 10.1093/protein/gzs024.

[12] V. Kunik, S. Ashkenazi, and Y. Ofran, "Paratome: An online tool for systematic identification of antigen-binding regions in antibodies based on sequence or structure," Nucleic Acids Res., vol. 40, no. W1, pp. 521-524, 2012, doi: 10.1093/nar/gks480.

[13] P. P. Olimpieri, A. Chailyan, A. Tramontano, and P. Marcatili, "Prediction of site-specific interactions in antibody-antigen complexes: The proABC method and server," Bioinformatics, vol. 29, no. 18, pp. 2285-2291, 2013, doi: 10.1093/bioinformatics/btt369.

[14] F. Ambrosetti et al., "proABC-2: PRediction Of AntiBody Contacts v2 and its application to information-driven docking," Bioinformatics, no. July, pp. 1-2, 2020, doi: 10.1093/bioinformatics/btaa644.

[15] E. Liberis, P. Velickovic, P. Sormanni, M. Vendruscolo, and P. Lio, "Parapred: Antibody paratope prediction using convolutional and recurrent neural networks," Bioinformatics, vol. 34, no. 17, pp. 2944-2950, 2018, doi: 10.1093/bioinformatics/bty305.

[16] A. Deac, P. Velickovic, and P. Sormanni, "Attentive Cross-Modal Paratope Prediction," J. Comput. Biol., vol. 26, no. 6, pp. 536-545, 2019, doi: 10.1089/cmb.2018.0175.

[17] V. Kunik, B. Peters, and Y. Ofran, "Structural consensus among antibodies defines the antigen binding site.," PLoS Comput. Biol., vol. 8, no. 2, p. e1002388, 2012, doi: 10.1371/journal.pcbi.1002388.

[18] S. Lu, Y. Li, F. Wang, X. Nan, and S. Zhang, "Leveraging Sequential and Spatial Neighbors Information by Using CNNs Linked With GCNs for Paratope Prediction," IEEE/ACM Trans. Comput. Biol. Bioinforma., pp. 1-1, 2021, doi: 10.1109/TCBB.2021.3083001.

[19] A. Del Vecchio, A. Deac, P. Liò, and P. Veličković, "Neural message passing for joint paratope-epitope prediction," arXiv, pp. 1-5, 2021, [Online]. Available: http://arxiv.org/abs/2106.00757.

[20] S. Pittala and C. Bailey-Kellogg, "Learning context-aware structural representations to predict antigen and antibody binding interfaces," Bioinformatics, vol. 36, no. 13, pp. 3996-4003, 2020, doi: 10.1093/bioinformatics/btaa263.

[21] S. Daberdaku and C. Ferrari, "Antibody interface prediction with 3D Zernike descriptors and SVM," Bioinformatics, vol. 35, no. 11, pp. 1870-1876, 2018, doi: 10.1093/bioinformatics/bty918.

[22] M. Zeng, F. Zhang, F. X. Wu, Y. Li, J. Wang, and M. Li, "Proteinprotein interaction site prediction through combining local and global features with deep neural networks," Bioinformatics, vol. 36, no. 4, pp. 1114-1120, 2020, doi: 10.1093/bioinformatics/btz699.

[23] L. Guo et al., "DeepPSP: A Global-Local Information-Based Deep Neural Network for the Prediction of Protein Phosphorylation 
Sites," J. Proteome Res., vol. 20, no. 1, pp. 346-356, 2021, doi: 10.1021/acs.jproteome.0c00431.

[24] J. P. A. Vieira and R. S. Moura, "An analysis of convolutional neural networks for sentence classification," in Conference on Empirical Methods in Natural Language Processing, 2017, vol. 2017-Janua, pp. 1-5, doi: 10.1109/CLEI.2017.8226381.

[25] J. Hu, L. Shen, S. Albanie, G. Sun, and E. Wu, "Squeeze-andExcitation Networks," IEEE Trans. Pattern Anal. Mach. Intell., vol. 42, no. 8, pp. 2011-2023, 2020, doi: 10.1109/TPAMI.2019.2913372.

[26] S. Hochreiter and J. Schmidhuber, "Long Short-Term Memory," Neural Comput., vol. 9, pp. 1735-1780, 1997.

[27] P. Zhou et al., "Attention-based bidirectional long short-term memory networks for relation classification," in Annual Meeting of the Association for Computational Linguistics, 2016, pp. 207-212, doi: 10.18653/v1/p16-2034.

[28] Y. Bin, Y. Yang, F. Shen, N. Xie, H. T. Shen, and X. Li, "Describing video with attention-based bidirectional LSTM," IEEE Trans. Cybern., vol. 49, no. 7, pp. 2631-2641, 2019, doi: 10.1109/TCYB.2018.2831447.

[29] K. Krawczyk, T. Baker, J. Shi, and C. M. Deane, "Antibody iPatch prediction of the antibody binding site improves rigid local antibody-antigen docking," Protein Eng. Des. Sel., vol. 26, no. 10, pp. 621-629, 2013, doi: 10.1093/protein/gzt043.

[30] S. Ferdous and A. C. R. Martin, "AbDb: antibody structure database-a database of PDB-derived antibody structures," Database, vol. 2018, pp. 1-9, Jan. 2018, doi: 10.1093/database/bay040.

[31] J. Ren, Q. Liu, J. Ellis, and J. Li, "Tertiary structure-based prediction of conformational B-cell epitopes through B factors," Bioinformatics, vol. 30, no. 12, pp. 264-273, 2014, doi: 10.1093/bioinformatics/btu281.

[32] W. Zhang, Y. Xiong, M. Zhao, H. Zou, X. Ye, and J. Liu, "Prediction of conformational B-cell epitopes from 3D structures by random forests with a distance-based feature," BMC Bioinformatics, vol. 12, no. 341, pp. 1-10, 2011, doi: 10.1186/1471-2105-12-341.

[33] A. K. C. C. Wong, H. Y. Sze-To, and G. L. Johanning, "Pattern to Knowledge: Deep Knowledge-Directed Machine Learning for Residue-Residue Interaction Prediction," Sci. Rep., vol. 8, no. May, pp. 1-14, 2018, doi: 10.1038/s41598-018-32834-z.

[34] Q. Hou, P. F. G. De Geest, W. F. Vranken, J. Heringa, and K. A. Feenstra, "Seeing the trees through the forest: Sequencebased homo- and heteromeric protein-protein interaction sites prediction using random forest," Bioinformatics, vol. 33, no. 10, pp. 1479-1487, 2017, doi: 10.1093/bioinformatics/btx005.

[35] J. Zhang, Z. Ma, and L. Kurgan, "Comprehensive review and empirical analysis of hallmarks of DNA-, RNA-and protein-binding residues in protein chains," Brief. Bioinform., vol. 20, no. 4, pp. 1250-1268, 2018, doi: 10.1093/bib/bbx168.

[36] J. Zhang and L. Kurgan, "SCRIBER: Accurate and partner typespecific prediction of protein-binding residues from proteins sequences," Bioinformatics, vol. 35, no. 14, pp. i343-i353, 2019, doi: 10.1093/bioinformatics/btz324.
[37] J. Hu, Y. Li, M. Zhang, X. Yang, H. Bin Shen, and D. J. Yu, "Predicting Protein-DNA Binding Residues by Weightedly Combining Sequence-Based Features and Boosting Multiple SVMs," IEEE/ACM Trans. Comput. Biol. Bioinforma., vol. 14, no. 6, pp. 1389-1398, 2017, doi: 10.1109/ТCBB.2016.2616469.

[38] K. Yan, J. Wen, Y. Xu, and B. Liu, "Protein Fold Recognition Based on Auto-Weighted Multi-view Graph Embedding Learning Model," IEEE/ACM Trans. Comput. Biol. Bioinforma., vol. 5963, no. c, pp. 1-1, 2020, doi: 10.1109/tcbb.2020.2991268.

[39] M. J. Skwark, D. Raimondi, M. Michel, and A. Elofsson, "Improved Contact Predictions Using the Recognition of Protein Like Contact Patterns," PLoS Comput. Biol., vol. 10, no. 11, pp. 1-14, 2014, doi: 10.1371/journal.pcbi.1003889

[40] S. F. Altschul et al., "Gapped BLAST and PSI-BLAST: a new generation of protein database search programs," Nucleic Acids Res., vol. 25, no. 17, pp. 3389-3402, 1997.

[41] S. McGinnis and T. L. Madden, "BLAST: At the core of a powerful and diverse set of sequence analysis tools," Nucleic Acids Res., vol. 32, no. WEB SERVER ISS., pp. 20-25, 2004, doi: $10.1093 /$ nar/gkh435.

[42] J. Meiler, M. Müller, A. Zeidler, and F. Schmäschke, "Generation and evaluation of dimension-reduced amino acid parameter representations by artificial neural networks," J. Mol. Model., vol. 7, no. 9, pp. 360-369, 2001, doi: 10.1007/s008940100038.

[43] M. S. Klausen et al., "NetSurfP-2.0: Improved prediction of protein structural features by integrated deep learning," Proteins Struct. Funct. Bioinforma., vol. 87, no. 6, pp. 520-527, 2019, doi: $10.1002 /$ prot.25674.

[44] W. Wardah et al., "Predicting protein-peptide binding sites with a deep convolutional neural network," J. Theor. Biol., vol. 496, p. 110278, Jul. 2020, doi: 10.1016/j.jtbi.2020.110278.

[45] M. Ragoza, J. Hochuli, E. Idrobo, J. Sunseri, and D. R. Koes, "Protein-Ligand Scoring with Convolutional Neural Networks," J. Chem. Inf. Model., vol. 57, no. 4, pp. 942-957, 2017, doi: 10.1021 /acs.jcim.6b00740.

[46] M. Karimi, D. Wu, Z. Wang, and Y. Shen, "DeepAffinity: Interpretable deep learning of compound-protein affinity through unified recurrent and convolutional neural networks," Bioinformatics, vol. 35, no. 18, pp. 3329-3338, 2019, doi: 10.1093/bioinformatics/btz111.

[47] L. Luo et al., "An attention-based BiLSTM-CRF approach to document-level chemical named entity recognition," Bioinformatics, vol. 34, no. 8, pp. 1381-1388, 2018, doi: 10.1093/bioinformatics $/$ btx761

[48] L. Li, J. Wan, J. Zheng, and J. Wang, "Biomedical event extraction based on GRU integrating attention mechanism," BMC Bioinformatics, vol. 19, no. Suppl 9, pp. 93-100, 2018, doi: 10.1186/s12859018-2275-2.

[49] A. Fout, J. Byrd, B. Shariat, and A. Ben-Hur, "Protein interface prediction using graph convolutional networks," in Conference on Neural Information Processing Systems, 2017, vol. 30, no. Nips, pp. $6531-6540$. 
bioRxiv preprint doi: https://doi.org/10.1101/2021.07.17.452531; this version posted July 19, 2021. The copyright holder for this preprint (which was not certified by peer review) is the author/funder, who has granted bioRxiv a license to display the preprint in perpetuity. It is made available under aCC-BY-NC-ND 4.0 International license.

[50] L. A. Staeheli and D. Mitchell, "The Relationship Between Precision-Recall and ROC Curves Jesse," in International Conference on Machine Learning, 2006, pp. 233-240, doi: 10.1145/1143844.1143874.

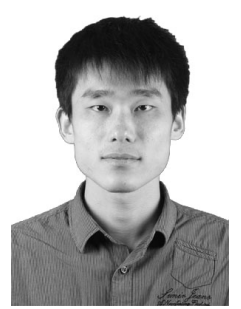

Shuai Lu received the BS degree from the School of Information Engineering of Zhengzhou University, Zhengzhou, Henan, China in 2014. Currently, he is working towards the PhD degree in the School of Information Engineering of Zhengzhou University. His current research interests mainly include machine learning and data mining applied to the biological field, especially in the prediction of protein-protein and antibody-antigen interactions.

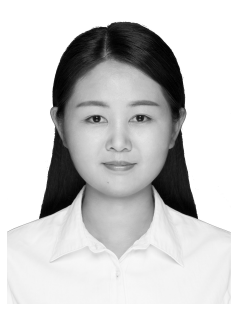

Yuguang Li received the MS degree from the School of Information Engineering, Zhengzhou University, Zhengzhou, Henan, China in 2018. Her research interests include bioinformatics, data mining, and NLP.

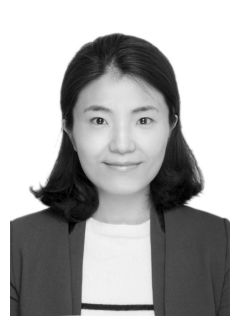

Xiaofei Nan received the PhD degree from the University of Mississippi. She is a vice professor in the School of Information Engineering, Zhengzhou University, Henan. Her current interests include pattern recognition, data mining, and bioinformatics.

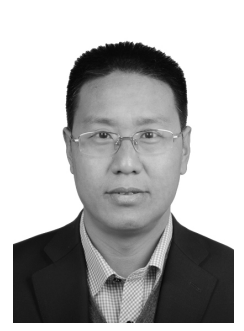

Shoutao Zhang received the $\mathrm{PhD}$ degree from the Northwest A\&F University. He has published about 40 high-quality referred papers in international conferences and journals. He is a professor in the School of Life Sciences, Zhengzhou University, Henan. His research interests include bioinformatics. 